Originalveröffentlichung in: Hausmann, Andrea (Hrsg.): Handbuch Kunstmarkt : Akteure, Management und Vermittlung, Bielefeld 2014, S. 235-250 (Schriften zum Kultur- und Museumsmanagement)

\title{
Zwischen Markt und Wissenschaft: Kunstsachverständige und Experten
}

Nils Büttner/Behrend Finke

\section{Eine Art Einleitung}

Es war ein veritabler Skandal, der nicht nur in Berlin die Wogen hochgehen ließ. ${ }^{1}$ Im August des Jahres 1671 hatte Friedrich Wilhelm von Brandenburg, der Große Kurfürst, eine von Generalfeldmarschall Alexander Freiherr von Spaen geleitete Delegation in die Niederlande gesandt. Neben der Aushandlung eines Bündnisses gegen Frankreich war sie beauftragt, Kunstwerke für die Berliner Sammlung zu erwerben. Der angesehene Amsterdamer Kunsthändler Gerrit Uylenburgh, den man zu diesem Zweck aufgesucht hatte, konnte gleich mit zwölf Werken von Michelangelo, Raffael und Tizian aufwarten. Da italienische Werke von berühmten Malern der Renaissance bislang in der kurfürstlichen Sammlung kaum vertreten waren, kam man ins Geschäft. Der Kaufpreis von etwas mehr als 2.000 Gulden war für ein Dutzend Gemälde von so berühmten Meistern erstaunlich niedrig. ${ }^{2}$ Schließlich hatte ein einzelnes Porträt Raffaels, das Bildnis des Baldassare Castiglione, bei seiner Versteigerung in Amsterdam im April 1639 schon 3.500 Gulden erzielt. ${ }^{3}$ Dass dem Großen Kurfürsten das Bildnis eines alten Mannes von der Hand Raffaels nun für nur 150 Gulden angeboten wurde, mag den Verdacht des am Berliner Hof tätigen Malers Hendrick Fromantiou geweckt haben. Der hatte vordem selbst als Angestellter in Uylenburghs Bilderfabrik gearbeitet und dort wie am Fließband Bilder kopiert. Uylenburgh handelte nämlich nicht nur mit Kunst,

1 | Friso Lammertse: Gerrit Uylenburgh, art dealer and painter in Amsterdam and London, in: Uylenburgh \& Son: Art and commerce from Rembrandt to De Lairesse 1625-1675, Ausstellungskatalog: London, Dulwich Picture Gallery, 7. Juni - 3. September 2006; Amsterdam, The Rembrandt House Museum, 16. September - 10. Dezember 2006, hg. von Friso Lammertse, Zwolle 2006, S. 61-114, zu dem hier dargestellten Fall bes. S. 79-91.

2 | Lammertse 2006 (wie Anm. 1), S. 82 f.

3 | The Rembrandt documents, hg. von Walter L.Strauss, Marjon van der Meulen, unter Mitarbeit von S.A.C. Dudok van Heel und P.J.M. de Baar, New York 1979, Nr. 1639/8, S. 177. 
er ließ sie auch marktgerecht produzieren. Da Fromantiou selbst bei Uylenburgh »auf der Galeere gesessen« hatte, wie man in Künstlerkreisen das Schicksal angestellter Maler wenig schmeichelhaft umschrieb, warnte er den Kurfürsten eindringlich vor den Geschäftspraktiken seines einstigen Chefs. ${ }^{4}$ Der Kurfürst sandte daraufhin seinen Maler nach Amsterdam, um den Beweis für seine Anschuldigung zu erbringen. In Amsterdam setzte Uylenburgh alles daran, die Echtheit der Bilder zu beweisen. In Gegenwart eines Notars wurden Amsterdamer Maler in das Gasthaus De Keijserskroon in der Kalverstraat bestellt, die über die dort ausgestellten Werke urteilen sollten. Selbst der Magistrat wurde eingeschaltet, weil der aus Berlin angereiste Maler die Unabhängigkeit der vom Kunsthändler Uylenburgh aufgebotenen Gutachter bezweifelte. Im Großen und Ganzen fiel diese Beurteilung durch Uylenburghs Amsterdamer Kollegen tatsächlich positiv aus und sie bescheinigten ihm, echte und qualitätvolle italienische Bilder verkauft $\mathrm{zu}$ haben, wobei nur wenige soweit gingen, auch die Zuschreibungen für zutreffend zu erklären. Im Wissen um Uylenburghs Einfluss innerhalb der Amsterdamer Lukas-Gilde, dem Zusammenschluss der Maler und Kunsthändler der Stadt, war Fromantiou nicht geneigt, die Sache damit auf sich beruhen zu lassen. Auch sein Ruf als Experte stand auf dem Spiel und so ließ er die Bilder in Den Haag durch weitere als Experten hinzugezogene Maler begutachten. Neben den in Den Haag tätigen Malern wurden sogar Gutachter aus Antwerpen und Rotterdam zu Rate gezogen. Aus dem nahegelegenen Delft waren Johannes Jordaens und Jan Vermeer angereist, deren Urteil vernichtend ausfiel. Es seien »nicht nur keine hervorragenden italienischen Gemälde, sondern im gegenteil großer Müll und schlechte Malereien, die kaum den zehnten Teil des verlangten Preises wert « seien. ${ }^{5}$ An eine Lieferung der Bilder nach Berlin war danach nicht mehr zu denken. Uylenburgh erhielt die Bilder zurück, um sie anderweitig zu vermarkten. Stets betonte er, nach bestem Wissen und Gewissen gehandelt zu haben. Der Maler und Kunstschriftsteller Arnold Houbraken berichtete noch 1719 von der seinerzeit vielbeachteten

4 I Houbraken, Arnold: De Groote Schouburgh der Nederlantsche Konstschilders en Schilderessen, 3 Bde., Amsterdam 1718-1721, hier: Bd. 2, S. 294f.: "Deze die voor henen op de Galey (gelykmen Italien het schilderen voor de keelbeulen dus gewoon is te noemen) gezetten, en zelf voor Uilenburg geschilderd had, en dus den handel dier vosschen kende, doopte de zelve met den naam van kopyen. " ["Dieser, der ehedem selbst auf der Galeere gesessen hatte (wie man in Italien das Malen für diese Halsabschneider gewöhnlich nennt) und selbst für Uylenburgh gemalt hatte und deshalb den Handel dieses Fuchses kannte. Er taufte sie auf den Namen Kopien."]

5 | Montias, John Michael: Vermeer en zijn milieu, Baarn 1993, Nr. 341, S. 380: " Niet alleen niet en syn uytmundende Italiaense Schilderiën, maer ter contrarie eenige groote rodden ende slechte schilderiën, die op verre nae de tiende part van de voosz. uytgetrocke prysen niet geacht en konnen werden. " [Nicht allein sind das keine hervorragenden italienischen Gemälde, sondern im Gegenteil ein großer Müll und schlechte Bilder, die weit entfernt davon sind für ein zehntel des angesetzten Preises geschätzt zu werden. "] 
Affäre, derzufolge Uylenburgh es verabsäumte, dem Kurfürsten den durch seinen Unterhändler geleisteten Vorschuss von 2.000 Reichstalern zurückzuerstatten. ${ }^{6}$

\section{Damals Und heute}

Diese ausführlich dokumentierte Begebenheit aus dem 17. Jahrhundert zeigt, dass Kunst schon immer eine sensible Ware war, deren Geldwert erheblich vom sachverständigen Urteil abhängt. Zugleich enthält die kleine Geschichte alles, was noch heute einen guten Kunstmarktkrimi auszeichnet. Dazu gehören neben dem Sammler, der selbst kein Experte ist, der Händler, der gleichermaßen Kunst und Expertise liefert und Sachverständige, die aufgefordert sind, die Sache unabhängig einzuschätzen und zu bewerten. Wie im Europa jener Jahre allgemein üblich, gehörten die Sachverständigen der Gilde an, die auf das entsprechende Handwerk und Gewerbe ein Privileg hatte. Die Maler und die seit dem späten 16. Jahrhundert als eigener Berufszweig greifbaren Kunsthändler waren dabei in einer Gilde zusammengeschlossen. In katholischen Gegenden war sie zugleich eine spirituelle Gemeinschaft, die im Laufe des Kirchenjahres vor allem religiöse und repräsentative Aufgaben erfüllte und in der Regel eine eigene, dem hl. Lukas geweihte Kapelle unterhielt. Allerdings war die Maler-Gilde nicht in allen Städten eine unter dem $\mathrm{Pa}$ trozinium des hl. Lukas zusammengeschlossene Vereinigung von künstlerischen Gewerben. Das ließ den Maler und Kunstschriftsteller Karel van Mander 1604 über die »allzu undankbare[n] Jahrhunderte der Gegenwart« klagen, »in denen man auf Drängen gemeiner Pfuscher solch schändlichen Gesetzen und mißgünstigen Verordnungen in den Städten Geltung verschafft hat, so daß fast überall (Rom fast allein ausgenommen) aus der edlen Malkunst eine Gilde gemacht wird, wie man es mit allen rohen Handwerken und Gewerben, als da sind: Weben, Pelznähen, Zimmern, Schmieden und dergleichen tut. Zu Brügge in Flandern bilden die Maler nicht allein eine Gilde, sondern es gehören auch noch die Pferdegeschirrmacher dazu. Zu Haarlem, wo es stets viele hervorragende Maler gegeben hat, gehören die Kesselflicker, Zinngießer und Trödler zur Malergilde. Obwohl diese beiden Städte Gründe dafür geltend machen, warum dies so geschah, ist es doch so weit gekommen, dass man fast gar keinen Unterschied zwischen Malen und Schuhflicken, Weben und dergleichen Dingen macht; denn es muss - wie Unwissenheit und Unverstand es gern haben - in eine Gilde gezwängt werden, und die Erlaubnis zu seiner Ausübung muss - dort wo man sie noch erlangen kann - mit Geld erkauft werden. ${ }^{7}$ Allen Klagen zum Trotz endeten Zunftzwang und Konzessionswe-

6 | Houbraken 1718-21 (wie Anm. 4), Bd. 2, S. 295.

7 | Van Mander; Carel: Das Leben der niederländischen und deutschen Maler des Carel van Mander, Textabdruck nach der Ausgabe von 1617, übers. u. Anmerkungen von Hanns Floerke, Bd. 1, München 1906, S. S. 387-389; [Van Mander, Karel]: Het Schilder-Boeck, Haarlem 1604 , fol. $241 v-252 r$. 
sen in den meisten europäischen Ländern erst im Verlauf des 19. Jahrhunderts. In Frankreich wurde in Folge der Revolution 1791 die Gewerbefreiheit eingeführt, in Preußen 1810 , in Württemberg $1862 .{ }^{8}$ Im Zuge der damit einhergehenden Neuregelungen wurden Gewerbeordnungen erlassen, in denen auch das Sachverständigenwesen geregelt wurde. Die am 21. Juni 1869 für den Norddeutschen Bund erlassene Ordnung, deren Geltung nach 1871 in die Gesetzgebung des Deutschen Reiches einging, wurde zum Vorläufer der noch heute gültigen Gewerbeordnungen. In ihr wurde erstmals die Anstellung qualifizierter Sachverständiger systematisch geregelt. Dafür waren in Preußen die kaufmännischen Korporationen zuständig gewesen, später im Deutschen Reich die Industrie- und Handelskammern, die als »Organe der wirtschaftlichen Selbstverwaltung den spezifischen Bedürfnissen des Wirtschaftsverkehrs Rechnung tragen sollten. ${ }^{9}$ Dazu gehörte einerseits eine möglichst große Freiheit des Handels, andererseits die notwendige Kontrolle, um die Allgemeinheit vor unsittlichem Handel und wirtschaftlicher Schädigung zu schützen. Seit jener Zeit gibt es öffentlich bestellte, vereidigte Sachverständige, die als unabhängige Gutachter die Güterproduktion, Handwerk, Handel und Gewerbe bewerten. Sachverständige wurden seit jener Zeit nicht nur im Wirtschaftsverkehr unabdingbar, sondern auch zu einer unentbehrlichen Instanz des Rechtslebens. Heute würde kein Richter mehr einen Verkehrsunfall oder einen Bauprozess entscheiden, ohne Sachverständige hinzuzuziehen, deren Gutachten der Feststellung und Beurteilung von Sachverhalten dienen. Doch was im Wirtschafts- und Rechtsleben so unverzichtbar wie selbstverständlich scheint, erweist sich zumal aus juristischer Perspektive - in Hinblick auf den Kunstmarkt als irritierend anders. Wie schon bei der einleitend erwähnten Auseinandersetzung, in deren Verlauf der Kunsthändler Uylenburgh etliche Maler als Gutachter aufbot, die bei ihm angestellt waren, ist die Unabhängigkeit der Gutachter auf dem Kunstmarkt bis heute keine Selbstverständlichkeit. So hat beispielsweise das Kölner Oberlandesgericht 2005 in einer Entscheidung festgestellt, dass die Bewertungspraxis von Kunstwerken durch die Häuser Christie's und Sotheby's akzeptabel wäre, da Kunst prinzipiell schwierig zu bewerten sei und beide Häuser über große Erfahrung verfügten. ${ }^{10} \mathrm{Um}$ die juristische Problematik dieser Feststellung zu verdeutlichen, wurde von kritischen Kommentatoren darauf verwiesen, dass im Falle des steuerlichen Wertansatzes

8 I Desch, Eberhard: Die öffentliche Bestellung des Sachverständigen. Zur Geschichte des Sachverständigenwesens, in: Der Sachverständige 24, 1997, S. 7-10; Stieglitz, Leo von: Zünfte in Württemberg: Regeln und Zeichen altwürttembergischer Zünfte vom 16. bis zum 19. Jahrhundert, Stuttgart 2000, S. 119.

9 | Desch 1997 (wie Anm. 8), S. 9, vgl. auch Konstantinou, Konstantinos: Die öffentliche Bestellung von Sachverständigen nach § 36 Gew0, Köln 1993.

10 | Oertzen, Christian von: Anmerkung zum Urteil des OLG KöIn vom 05.10.2005, Az.: 2 U 153/04 (Erfüllung eines Wertermittlungsanspruchs bzgl. Kunstgegenständen durch Bewertungen von Sotheby's und Christie's), in: Zeitschrift für Erbrecht und Vermögensnachfolge 13,2006, S. $77-80$. 
einer Immobilie, des Zugewinnausgleichs oder von Pflichtteilsansprüchen, wohl keinesfalls das Gutachten eines aktiv am Verkauf beteiligten Immobilienmaklers zur Begründung dienen würde, das sich auf die lapidare Feststellung beschränkt, der Wert der Immobilie liege zwischen fünf und sieben Millionen Euro. ${ }^{11}$ Weder eine Finanzbehörde noch ein Gericht würden eine solche Bewertung akzeptieren, deren Grundlage genauso diffus bleibt, wie der Weg ihrer Ermittlung. Auf dem Kunstmarkt sind derartige Bewertungen durch Auktionshäuser und den Handel gängige Praxis, wobei die Expertise kein Privileg von Auktionatoren oder Händlern ist, aber eben auch nicht von unabhängigen Experten oder Sachverständigen. Zwischen beiden Gruppen muss unterschieden werden.

\section{EXPERTEN}

Als Experten treten auf dem Feld der Kunst zahlreiche Personen und Gremien auf, die zu einem bestimmten Künstler, zu einem Werk oder einem Euvre, zu einer Epoche oder Kunstlandschaft besonderen Sachverstand erlangt haben oder über Fachkunde verfügen. Zumeist sind sie über wissenschaftliche Forschungsleistungen oder über langjährige praktische Erfahrung ausgewiesen. Der Begriff des »Experten « ist dabei weder eine geschützte Berufsbezeichnung noch an eine spezifische Prüfung der Eignung oder formalisierte Kriterien der Ernennung geknüpft. ${ }^{12}$ Wer als Experte gilt, wird durch ein komplexes Gefüge diskursiver Praktiken bestimmt. Dabei sind die Resultate dieser Diskurse leichter zu beschreiben als ihre inneren Wirkmechanismen. ${ }^{13}$ Ohne selbst den Wert einzuschätzen, nehmen Experten unmittelbar auf die wertbildenden Faktoren des Kunstmarktes Einfluss, indem sie sich beispielsweise öffentlich zu Echtheit und Erhaltungszu-

11 I Heuer, Carl-Heinz: Die Bewertung von Kunstwerken im Steuer- und Erbrechtsstreit, in: Kunst im Markt - Kunst im Recht: Tagungsband des III. Heidelberger Kunstrechtstags am 09. und 10. Oktober 2009, hg. von Thomas Dreier, Nicolai Kemle, Peter M. Lynen, Matthias Weller, Baden-Baden 2010, S. 121-127, hier: S. 121.

12 | Brühl, Friederike von: Marktmacht von Kunstexperten als Rechtsproblem: Der Anspruch auf Erteilung einer Expertise und auf Aufnahme in ein Werkverzeichnis, KöIn [u.a.] 2008, S. 24f.; Gerlach, Tilo: Die Haftung für fehlerhafte Kunstexpertisen, Baden-Baden 1998, S. 33; Simons, Simone: Fachliche Autorität im Kunsthandel und ihre haftungsrechtliche Bedeutung, Konstanz 1999, S. 3; Duret-Robert, Francois: Droit du marché de l'art, Paris 2007, S. 171.

13 | Michel Foucault der mit seinem Diskurs-Begriff das enge Beziehungsgefüge beschrieb, das zwischen "Institutionen, ökonomischen und gesellschaftlichen Prozessen, Verhaltensformen, Normsystemen, Techniken, Klassifikationstypen und Charakterisierungsweisen "Verbindungen herstellt, sagt leider nicht, wie Diskurse dingfest gemacht werden können. Vgl. Foucault, Michel: Archäologie des Wissens (1969), Frankfurt a.M. 1997, S. 68 . 
stand oder zu Fragen der Eigenhändigkeit oder Datierung von auf dem Markt verfügbaren Werken der bildenden Kunst äußern. Die Zuschreibungsautorität einzelner Experten ist dabei ein für den Kunstmarkt spezifisches Phänomen, zu dem inzwischen eine umfangreiche Spezialliteratur existiert. ${ }^{14}$

Experten und Sachverständige fundieren und stabilisieren das Glaubensuniversum, das den Geldwert eines Kunstwerkes bestimmt. Gerade bei hochpreisig gehandelten Künstlern und Werken ist dabei zu bemerken, dass »jeder Künstler seinen Experten und jeder Experte seinen Künstler hat«. ${ }^{15}$ Die anerkannten Experten für bestimmte Künstler haben damit die Macht, Werte zu schaffen oder zu vernichten. ${ }^{16}$ Entsprechend ist es den Eigentümern und Händlern von Kunstwerken ein Anliegen, dass ihre Werke von den entsprechenden Experten für den jeweiligen Künstler akzeptiert und in das Euvreverzeichnis aufgenommen werden. Die Zuschreibungsautorität eines Experten ist dabei keine Frage der beruflichen Vorbildung sondern zumeist das Resultat einer langen und intensiven Beschäftigung mit dem jeweiligen Spezialgebiet. So galt etwa der praktizierende Mediziner Hans-Ulrich Beck als führender Experte für das Werk des niederländischen Landschaftsmalers Jan van Goyen, dessen umfangreiches E Euvre er in einem mehrbändigen Catalogue Raisonné verzeichnet hatte. ${ }^{17}$ "Die echte Kennerschaft«, hatte Max J. Friedländer 1919 proklamiert, »ist Spezialistentum. Es gibt keine Universalkenner «. ${ }^{18}$ Dieser Satz hat tatsächlich noch heute Gültigkeit, auch wenn die Kennerschaft zu bestimmten Sachgebieten von einem Expertengremium oder einem Zuschreibungskomitee vertreten wird. Ein Beispiel dafür ist das 1990 ins Leben gerufene »Pollock-Krasner Authentication Board«, das die Echtheit der vielgefälschten Werke von Jackson Pollock und seiner Witwe Lee Krasner zertifiziert. ${ }^{19}$

Dass die Echtheit eines Werkes auf dem Kunstmarkt zu den gewichtigsten wertbildenden Faktoren zählt, ist eine banale Feststellung. ${ }^{20}$ Sie ist aber von großer Tragweite für die Bedeutung der Experten. Die Echtheit eines Werkes wird nämlich nicht nur durch dessen materielle Substanz garantiert, sondern stets

14 | Brühl 2008 (wie Anm. 12), S. 47-60, mit weiterer Literatur; Simons (wie Anm. 12).

15 | Simons (wie Anm. 12), S. 15.

16 | Friedländer, Max J.: Der Kunstkenner, Berlin 1919, S. 8.

17 | Beck, Hans-Ulrich: Jan van Goyen: 1596-1656: Ein Oeuvreverzeichnis, 4 Bde., Amsterdam 1972-1991. Zur Biografie vgl. Geoge Keyes: Hans-Ulrich Beck (1930-2010), in: Historians of Netherlandish Art Newsletter, April 2011, S. 4.

18 | Friedländer, Max J.: Der Kunstkenner, Berlin 1919, S. 11.

19 | Ähnliche Komitees wurden für das CEuvre von Andy Warhol und Jean-Michel Basquiat eingerichtet. Vgl. Spencer, Ronald D.: The expert versus the object: Judging fakes and false attributions in the visual arts, Oxford [u.a.] 2004, S. 21.

20 | Zur diskursiven Geschichte der Idee von Original und Kopie vgl. Déjà-vu? Die Kunst der Wiederholung von Dürer bis YouTube, Ausstellungskatalog: Staatliche Kunsthalle Karlsruhe, hg. von Ariane Mensger, Bielefeld 2012. 
auch durch das Urteil von Experten. Es gibt inzwischen eine große Zahl kunsttechnologischer Methoden der Echtheitsprüfung von Kunstwerken, die zur Grundlage von Zuschreibungen werden. ${ }^{21}$ Doch liefert auch die genaueste technische Untersuchung dabei nur selten unbestreitbare Hinweise auf die Autorschaft eines bestimmten Künstlers. So kann die technische Untersuchung und chemische Analyse von Malmaterialien zwar den Nachweis erbringen, dass ein Werk erst zu einem bestimmten historischen Zeitpunkt möglich war, doch über die Zuschreibung an einen Künstler ist damit nichts ausgesagt. Eine kunsttechnologische Untersuchung erbrachte zum Beispiel den Beweis, dass in einem vom Kölner Auktionshaus Lempertz im Jahr 2006 als Werk des rheinischen Expressionisten Heinrich Campendonk versteigerten Gemälde Titanweiß Verwendung gefunden hatte. ${ }^{22}$ Dieses Pigment war aber zur angeblichen Entstehungszeit des Bildes im Jahr 1914 noch gar nicht erfunden. Damit ließ sich der Nachweis führen, dass es sich bei dem Bild um eine Fälschung handelte. ${ }^{23}$ Kurz zuvor hatte im Auftrag des Auktionshauses die Verfasserin des Werkverzeichnisses von Heinrich Campendonk ein Gutachten erstellt, in dem sie eine kunsttechnologische Analyse empfahl. ${ }^{24}$ Nachdem die Fälschung aufgeflogen war, verklagte die maltesische Firma Trasteco Ltd., die den gefälschten Campendonk ersteigert hatte, das Kölner Auktionshaus auf Schadensersatz und Rückzahlung des Kaufpreises von 2,9 Millionen Euro. ${ }^{25}$ Das Argument des Auktionshauses, selbst vom Fälscher getäuscht worden zu sein, ließ das Gericht nicht gelten. Die Firma Lempertz hatte nämlich zu keinem der von der Fälscherbande aus der fiktiven »Sammlung Jägers« zur Auktion eingereichten Werke vor der Versteigerung eine Expertise eingeholt. Unter Verweis auf seine Gutgläubigkeit erklärte Henrik Hanstein, persönlich haftender Gesellschafter des Auktionshauses, sich zum Opfer einer betrügeri-

21 | Picker, Günther: Fälscher, Diebe, Betrüger. Die Kehrseite des Kunst- und Antiquitätenmarkts (Schriftenreihe der Akademie der Bildenden Künste in Nürnberg, 7), München 1994; Ders.: Antiquitäten, Kunstgegenstände. Alles über Recht, Steuern, Versicherung, Augsburg 1996. Allgemein zum Problem der Fälschung vgl. Müller-Straten, Christian: Fälschungserkennung, München 2011.

22 | Zum vieldiskutierten Fall Beltracchi vgl. Timm, Tobias und Koldehoff, Stefan: Falsche Bilder, echtes Geld. Der Fälschungscoup des Jahrhunderts - und wer alles daran verdiente, Berlin 2012.

23 | Eine Untersuchung durch das Münchener Doerner Institut erbrachte im März 2008 den Beweis, dass Titanweiß verwandt worden war. Eine zweite kunsttechnologische Untersuchung bestätigte im Ende 2008 dieses Ergebnis. Darüber hinaus entlarvte der Flechtheim-Experte Ralph Jentsch einen rückseitig auf dem Gemälde angebrachten Aufkleber als Fälschung.

24 | Firmenich, Andrea: Heinrich Campendonk: 1889-1957. Leben und expressionistisches Werk mit Werkkatalog des malerischen Oeuvres, Recklinghausen 1989.

25 | Vgl. dazu das Urteil der 2. Zivilkammer des Landgerichts Köln (Vorsitzende Richterin: Sabine Kretzschmar, Az: 20 457/08). 
schen Bande, auf die »sogar Werner Spies« hereingefallen sei. ${ }^{26}$ Werner Spies, ehemals Direktor des Centre Pompidou in Paris und weltweit bekannter Experte für das CEuvre des Malers Max Ernst, war mehrfach als Gutachter und Vermittler von Werken aus der fiktiven »Sammlung Jägers« tätig. Spies erteilte den in den Kunstmarkt geschleusten Fälschungen den Ritterschlag, indem er rückseitig auf einem Foto vermerkte, dass das abgebildete Werk in das von ihm verantwortete Werkverzeichnis aufgenommen werde. ${ }^{27}$ Sein Expertenurteil ließ Spies sich üppig honorieren, indem er von der Fälscherbande für jedes verkaufte Bild, das er vermittelte, eine Provision von sieben bis acht Prozent des Verkaufserlöses erhielt. ${ }^{28}$ Damit nicht genug ließ er sich auch von den Weiterverkäufern eine Provision pro verkauftes Bild zahlen, über deren Höhe der inzwischen in Frankreich in einem anderen Fall rechtskräftig verurteilte Werner Spies schweigt. ${ }^{29}$ Bislang gibt es keine Gesetze, die eine verbindliche Trennung von Vermittler- und Gutachtertätigkeiten vorschreiben. Werner Spies hat beides gleichermaßen betrieben und beispielsweise als Kunstvermittler einhunderttausend Dollar dafür erhalten, dass er die Eigentümer eines vermeintlichen Originalgusses der Picasso-Bronze »Tête de Fernande « mit Pablo Picassos Sohn Claude Ruiz-Picasso zusammenbrachte. ${ }^{30}$ Er gilt als der Einzige, der Zertifikate über die Echtheit von Picassos ausstellen kann. Nachdem er am 19. Oktober 2004 die Echtheit der Arbeit testiert hatte, wurde auch er von den Eigentümern entlohnt. Für rund sechs Millionen Dollar wurde die Bronze, von der man inzwischen weiß, dass es sich um einen Nachguss handelt, danach an den Verleger Samuel Newhouse verkauft. Der vermeintlich originale Picasso ist mit dieser Erkenntnis kaum mehr wert als die Bronze, aus der er besteht, obwohl das Werk haargenau so aussieht wie das Original. Da bei einem Bronzeguss die Hand des Künstlers nicht unmittelbar im Spiel ist, gewinnt die marktbestimmende Frage der Echtheit hier eine gleichsam metaphysische Dimension. ${ }^{31}$

26 | Voss, Julia und Maak, Niklas: Als ich mich fand in einem dunklen Walde, URL: www. faz.net/aktuell/feuilleton/kunst/systemkrise-im-kunstmarkt-als-ich-mich-fand-ineinem-dunklen-walde-14744.html (10.07.2013).

27 | "Ich verwende stets dieselbe Formulierung", erklärt Spies, "die ich auf der Rückseite eines Fotos des betreffenden Werks niederschreibe: L'oeuvre reproduite va figurer dans le catalogue raisonée Max Ernst qui paraît sous ma direction (Das abgebildete Werk wird in das von mir verantwortete Werkverzeichnis aufgenommen) ". Vgl. Voss/Maak 2013 (wie Anm. 26).

28 | Auch in Deutschland ist unter Sachverständigen die Praxis verbreitet, die Honorierung von Wertgutachten mit einem Prozentsatz vom ermittelten Wert zu berechnen.

29 | URL: www.lemonde.fr/culture/article/2013/05/27/1-historien-d-art-werner-spiescondamne_3418072_3246.html (10.07.2013).

30 | URL: http://law.justia.com/cases/new-york/other-courts/2008/2008-28450.html (10.07.2013); Vgl. Voss/Maak 2013 (wie Anm. 26).

31 | Vgl. Voss/Maak 2013 (wie Anm. 26). 


\section{EXPERTISE}

Der Wert eines Kunstwerkes liegt nicht oder nur zu Teilen in dessen materieller Substanz, sondern eher schon in einer durch die Meinungen der Protagonisten des Kunstmarktes fundierten und produzierten Idee von Werthaltigkeit. Da der Prozess der Wertfindung einer der Annäherung ist und keinen Absolutheitsanspruch erheben kann, wird das Expertenurteil zum zentralen Faktor der Wertbestimmung. Dass die Zuschreibungsautorität monetisierbar ist, muss fast zwangsläufig zur Folge haben, dass bei dem auf Augenscheinnahme basierenden stilistischen Urteil von Experten oft nicht nur stilkritische Fragestellungen eine Rolle spielen.

Die Anforderungen an Form und Inhalt einer den Wert des Kunstwerkes beeinflussenden Expertise haben sich vor allem in den letzten Jahrzehnten stark gewandelt. ${ }^{32}$ Während der auch als »Vater der heutigen Kunstexpertise in Deutschland « bezeichnete Wilhelm von Bode $(1845-1929)$ sich zumeist noch damit beschied, auf leeren Zetteln oder auf der Rückseite von Fotografien zu vermerken, dass es sich seiner unverbrüchlichen Überzeugung nach um ein Werk von XY handele, sind die Anforderungen inzwischen stark gestiegen. In seiner Verteidigung machte zum Beispiel das Auktionshaus Lempertz geltend, dass noch um die Mitte der 199oer Jahre naturwissenschaftliche Untersuchungen nicht Standard gewesen seien. ${ }^{33}$ Gegenwärtig sind kunsttechnologische Gutachten neben der stilkritischen Analyse und der kunsthistorischen Einordnung tatsächlich im Bereich der hochpreisigen Kunst zur Regel geworden. Alte Gutachten und Zuschreibungen werden systematisch hinterfragt und manches sicher geglaubte Werk wird ab- oder neu zugeschrieben. Durch in großer Zahl ausgefertigte, allein stilkritisch argumentierende Gutachten hat manch ein Kunsthistoriker seinen Ruf nachhaltig geschädigt. Und die inzwischen historisch gewordenen Gutachten mancher Experten dokumentieren weniger die Werthaltigkeit eines Kunstwerkes als die diskursive Dynamik geisteswissenschaftlicher Erkenntnis.

Der Wandel in der Auffassung von den Möglichkeiten und Grenzen der Zuschreibung ist besonders an der Erforschung und Katalogisierung der Werke des niederländischen Malers Rembrandt ablesbar. Noch in der ersten Hälfte des 20. Jahrhunderts waren immer wieder neue Werke aufgetaucht und in die Cuvrekataloge aufgenommen worden. Als zu Rembrandts 300 . Geburtstag im Jubiläumsjahr 1906 in der Reihe »Klassiker der Kunst« die zweite Auflage des Kataloges von Adolph Rosenberg zu Rembrandts malerischem Werk erschien, waren darin 565 Bilder abgebildet. Die von Wilhelm R. Valentiner publizierte dritte Auflage aus

32 | Zum Rechtsbegriff der Expertise und den mit inm verbundenen Problemen vgl. Wolf, Andreas: Die Expertise. Inhalt, Form und Rechtswirkungen von Kunstgutachten, Dissertation, Bonn 2011, und die in Anm. 46 genannte Literatur.

33 | URL: www.spiegel.de/kultur/gesellschaft/beltracchi-affaere-auktionshaus-muss-mil lionen-schadensersatz-zahlen-a-858621.html (10.07.2013). 
dem Jahr 1909 enthielt 643 Werke, denen der Autor 1921 in einem Zusatzband noch einmal 120 »Wiedergefundene Gemälde« hinzufügte. Ihren Höhepunkt erreichte die Zahl der Zuschreibungen in den 1930er Jahren, als man Rembrandt mehr als 750 Gemälde zuschrieb. Die kritische kennerschaftliche Analyse von Abraham Bredius reduzierte diese Zahl 1935 auf nurmehr 630 Bilder, deren Zahl Horst Gerson 1968 in der Neuausgabe des bis heute unverzichtbaren Werkes auf 420 reduzierte. Damals hatte sich in Holland eine Gruppe von Forschern zusammengefunden, die auch mit Hilfe naturwissenschaftlicher Technologien zu einem objektiven und sachlich fundierten CEuvrekatalog gelangen wollten. Zwanzig Jahre lang hat dieses »Rembrandt Research Projekt« an den drei ersten, streng chronologisch geordneten Teilbänden gearbeitet. Danach übernahm das jüngste Mitglied dieser ersten Forschergruppe die Neukonzeption und legte 2005 einen thematisch geordneten Band zu den Selbstbildnissen vor, in den Werke als eigenhändig Aufnahme fanden, die in den ersten Teilbänden mit durchaus überzeugend anmutenden Argumenten abgeschrieben worden waren.

Gerade bei den $\mathrm{Zu}$ - und Abschreibungen alter Kunst ist man mit dem Problem konfrontiert, dass dem Namen eines Künstlers in der Vormoderne nicht die gleiche Bedeutung beigemessen wurde, wie das heute der Fall ist. Wie wenig die modernen Vorstellungen von gutem Geschmack, ästhetischer Raffinesse und künstlerischem Wert der allgemeinen Einschätzung der kunstbesitzenden Öffentlichkeit des 17. Jahrhunderts entsprachen, vermag ein Blick in zeitgenössische Inventare und Verzeichnisse des Kunstbesitzes zu erhellen. Kaum zehn Prozent der abertausenden von Gemälden, die damals in Nachlassinventaren verzeichnet wurden, waren einem Künstler zugeschrieben. Blickt man nun auf die Bestände der modernen Museen, sind es kaum zehn Prozent der Bestände, die nicht einem Künstler zugeschrieben werden. Mit Blick auf diese Zahlen will es beinahe scheinen, als würden im Laufe der Jahre die Informationen über einzelne Bilder und ihre Maler nicht etwa immer spärlicher fließen, sondern im Gegenteil immer reicher. Dabei ist jedoch durchaus anzunehmen, dass es damals ja sogar noch leichter gewesen sein dürfte, ein Bild einem bestimmten Maler zuzuweisen als es heute ist. Es lässt sich daraus also unschwer der Schluss ableiten, dass man sich für diese spezielle Information im Allgemeinen nicht in dem Maße interessierte, wie man dies heute tut.

Vor allem in den letzten Jahrzehnten haben das Interesse am jeweiligen Verfertiger eines Kunstwerkes und die Gesetzmäßigkeiten des Kunstmarktes, vor allem aber eine zunehmende Verknappung hochwertiger Werke namhafter Künstler zur Folge gehabt, dass inzwischen auch Maler über ein umfangreiches Geuvre verfügen, die man noch vor hundert Jahren kaum dem Namen nach kannte. Maler wie der auf Waldlandschaften spezialisierte Gillis van Coninxloo oder der Architekturmaler Hendrik van Steenwijk werden gleichsam unter einer Flut von Zuschreibungen schlechter Bilder erstickt. ${ }^{34}$ Gelingt es der kunsthistorischen 
Forschung den Kern eines Euvres aus dem Wust falscher Zuschreibungen herauszuschälen, dient dem Handel schon bald der Name eines anderen bislang wenig beachteten Künstlers als Sammelbecken. ${ }^{35}$ Und auch für diese Werke finden sich Kunden, da einer bislang nicht ernsthaft im Rückgang begriffenen Sammlerklientel immer weniger qualitätvolle Werke zur Verfügung stehen. Die zunehmende Verknappung hat ihren Grund darin, dass zumal hochkarätige Sammlungen durch Überführung in Stiftungen und Museen dem Handel dauerhaft entzogen werden. Je geringer die Zahl qualitätvoller Bilder namhafter Künstler ist, desto mehr gewinnen Experten und Sachverständige an Bedeutung, die den Wert der gehandelten Werke verbürgen müssen.

\section{SACHVERSTÄNDIGE}

Im allgemeinen Sprachgebrauch versteht man unter einem Sachverständigen »eine als unabhängige, überparteiliche und objektive Entscheidungsinstanz in Betracht kommende Person mit überdurchschnittlichen Fachkenntnissen auf einem Spezialgebiet« ${ }^{36}$ Die Begriffe »Experte« und »Sachverständiger werden oft synonym verwandt. Dennoch hat es sich zunehmend etabliert, die Verfasser von Werkverzeichnissen und spezifische Kenner eines eng umgrenzten Gebietes als Experten zu bezeichnen und den Begriff des Sachverständigen denjenigen vorzubehalten, die Wertgutachten erstellen und Echtheit, Marktwert und Sammelwürdigkeit von Kunstgegenständen taxieren und testieren. Der Begriff des »Sachverständigen « ist dabei nicht geschützt, so dass es aufgrund der in Deutschland geltenden Gewerbefreiheit jedem gestattet ist, als Sachverständiger aufzutreten und Gutachten zu erstellen. Nur »öffentlich bestellte und vereidigte Sachverständige« müssen eine besondere Qualifikation nachweisen und eine Sachkunde- und Eignungsprüfung ablegen, die in Deutschland von den Industrie- und Handelskammern organisiert wird. ${ }^{37}$ Ein $»$ öffentlich bestellter und vereidigter Sachverständiger« ist zu Objektivität, Unparteilichkeit und Weisungsfreiheit verpflichtet und macht sich bei einem Verstoß gegen diese Pflichten strafbar, genauso wer den Titel missbräuchlich verwendet. ${ }^{38}$

35 | Nachdem zum Beispiel eine ausführliche Monografie zu Frans Francken d.J. vorlag, wich der Handel in seinen Zuschreibungen auf unbekanntere Mitglieder der Malerfamilie Francken aus. VgI. Ursula Härting: Frans Francken der Jüngere (1581-1642): Die Gemälde mit kritischem Oeuvrekatalog, Freren 1989.

36 | Brühl 2008 (wie Anm. 12), S. 25.

37 | Die Bestellung eines Sachverständigen kann auch durch eine Handwerkskammer, eine Landwirtschaftskammer, eine Architekten-oder Ingenieurkammer oder durch das $\mathrm{Re}$ gierungspräsidium eines Landes erfolgen. VgI. URL: www.bv-kunstsachverstaendiger.de/; http://svv.ihk.de (10.07.2013).

38 | Vgl. § 132a StGB. 
Seit 1983 werden in Deutschland »öffentlich bestellte und vereidigte Sachverständige« nach der von einem Fachausschuss abgenommenen Eignungsprüfung nur noch für bestimmte Zeit und für ein fest umrissenes Sachgebiet bestellt. Die Regeln der Bestellung durch die Industrie- und Handelskammer sind in $\int 36$ der Gewerbeordnung festgelegt. ${ }^{39}$ Der Bereich »Kunstwerke und Antiquitäten« ist in fünf Hauptgebiete gegliedert:

1. Europäische ur- und vorgeschichtliche Gegenstände

2. Antike

3. Ostasien

4. Außereuropäische Kunstwerke ohne Ostasien

5. Nachantike europäische Kunstwerke

Der bei weitem umfassendste Bereich der europäischen Kunstwerke ist dann noch einmal in ${ }_{4}$ Sachgebiete unterteilt, die von »europäischen Gemälden bis ca. 1550 « über »Europäische Bücher und Druckgrafik bis 1900 « bis zu »Jugendstil/Art Deco « reichen. ${ }^{40}$ Wo es ehedem einen Sachverständigen für Kunst und Antiquitäten gegeben hatte, werden seit 1983 Experten für eng umgrenzte Spezialgebiete bestellt. Das hatte zur Folge, dass aus dem vorher umfassend tätigen Kunstsachverständigen ein Spezialist wurde bzw. werden sollte. Entsprechend reduzierten sich die ihn mit seinem Spezialgebiet betreffenden Aufträge, so dass manche auf den Kunsthandel oder auf einen anderen Brotberuf ausgewichen sind. Die von den Industrie- und Handelskammern geforderte wirtschaftliche Selbstständigkeit als Einzelunternehmer war und ist bei solchen Rahmenbedingungen in der Regel nicht erreichbar, und selbst dann, wenn sie gelingt, in wirtschaftlich schwierigen Zeiten kaum aufrechtzuerhalten. Außerdem löste diese Bestellungspraxis bei privaten Auftraggebern Befremden aus, da für die Begutachtung und Bewertung einer komplexen Sammlung eigentlich so viele Sachverständige wie Teilgebiete der Sammlung beauftragt werden müssten. Und bei gerichtlichen Aufträgen führt diese Praxis bei der Beauftragung durch das Gericht

39 | Vgl. §§ 36, 36a Gew0, Sachverständigenordnungen der IHKs. URL: www.stuttgart. ink24.de/linkableblob/2001724/.8./data/Sachverstaendigenordnung-data.pdf;jses sionid $=6$ F2BC2DC7B674FF88CE92ED3BF7AE0A0.repl2 (20.07.2013).

40 | "1. Europäische Gemälde bis ca. 1550, 2. Europäische Gemälde von 1550 bis 1800 , 3. Europäische Gemälde der Romantik und des Realismus (19. Jhd.), 4. Europäische Gemälde des Impressionismus und der klassischen Moderne (19./20. Jhd.), 5. Europäische zeitgenössische Gemälde des 20. Jhd., 6. Europäische Plastik bis 1550, 7. Europäische Plastik von 1550 bis 1800,8 . Europäische Plastik des 19. Jhd., 9. Europäische zeitgenössische Plastik, 10. Europäische Handzeichnungen bis 1550, 11. Europäische Handzeichnungen von 1550 bis 1800 , (...) 40. Europäische Ur- und Vorgeschichte, 41. Jugendstil/Art Deco." URL: www.ifsforum.de/download.php?sv \%5Bid \%5D =4647 (10.07.2013). 
und der Anerkennung durch die Parteien und ihrer Rechtsvertreter aus formalen Gründen ständig zu Problemen. ${ }^{41}$

Eng umgrenzte Fach- und Sachgebiete sind zwar die Voraussetzung einer vertieften Sachkenntnis, doch sind sie zugleich ein Hindernis für einen wirtschaftlich selbständig agierenden Sachverständigen, der darauf angewiesen ist, von seiner Expertise zu leben. Auf die erwähnte, durch Spezialisierungszwang herbeigeführte wirtschaftliche Schwächung der Kunstsachverständigen reagierten einige der potentiell Betroffenen indem sie sich - trotz entsprechender akademischer Bildung und fachlicher Eignung - nicht als Kunstsachverständige vereidigen ließen, sondern als Hausratsexperten. Ausweislich der Sachverständigenordnung für das Fachgebiet »Hausrat« verfügen die vereidigten Sachverständigen »um Einrichtungen und Gegenstände normal ausgestatteter Haushalte gutachtlich bewerten zu können«, über »umfassende Kenntnisse« über »Möbel [...] Teppiche, [...] Porzellan [...] Metalle « und »Kunst«, dabei beherrschen sie insbesondere die »Unterscheidung zwischen Originalen und Reproduktionen « und nehmen »Wertbestimmung anhand des Kunstpreisjahrbuchs und anderer Hilfsmittel« vor. Darüber hinaus müssen sie in vielen Bereichen über Grundkenntnisse verfügen, »um Abgrenzungs- und Echtheitsprobleme zu erkennen und rechtzeitig einen Spezialsachverständigen hinzuziehen zu können. $\star^{42}$ Nach Ansicht der Vertreter dieser Berufsgruppe gehören Kunstwerke und Antiquitäten zum gehobenen Hausrat und unterliegen somit ihrer Fachkompetenz, wobei sie in der Regel keinen »Spezialsachverständigen « hinzuziehen. Da sie der Spezialisierung nicht unterworfen sind, ist es ihnen vor diesem Hintergrund möglich, bei Auftragsverhandlungen eine Begutachtung und Bewertung aller vorhandenen Kunst- und Wertgegenstände aus einer Hand anzubieten, was den Auftraggebern verständlicherweise sehr entgegenkommt. Der Zuverlässigkeit der Expertise ist das aber so wenig zuträglich wie der Unabhängigkeit der Gutachter.

Die fortschreitende wirtschaftliche Schwächung der Kunstsachverständigen resultiert auch aus der Praxis führender Sachversicherungen, Kunsthistoriker für die Bewertung privaten Kunstbesitzes anzustellen sowie aus den Werbe- und Akquisitionsstrategien nahezu aller in Deutschland tätigen Auktionshäuser, die Eigentümer von Kunstwerken und Antiquitäten mit dem Versprechen kostenloser und unverbindlicher Schätzungen zur Einlieferung von Auktionsgut zu locken versuchen. Eine objektive Trennung des Sachverständigenwesens und der Gewinnerzielungsabsicht des Handels ist hierbei nicht immer gegeben. Dabei erweist sich auch die Tatsache als problematisch, dass weder die Sachverständigenordnung noch das Strafgesetzbuch den »öffentlich bestellten und vereidigten

41 | Deshalb gibt es seit 2012 eine Initiative unter Federführung des Instituts für Sachverständigenwesen e.V., die eine Neufestsetzung der fachlichen Bestellungsvoraussetzungen und Sachgebietseinteilungen zum Ziel hat.

42 | URL: www.ifsforum.de/dokumentdetail.php?sv[id]=4635 (10.07.2013). 
Sachverständigen« den Handel mit den Gegenständen ihrer Expertise ausdrücklich verbieten. ${ }^{43}$

\section{Risiken Und Haftung}

Gutachten und Expertisen sind längst nicht immer lukrativ und nur die wenigsten Experten für das Euvre eines bestimmten Künstlers ziehen wirtschaftlichen Profit aus ihrer spezifischen Kennerschaft. Denn zwar steigert das Vorliegen eines Werkkatalogs den materiellen Wert der darin erwähnten Werke, doch ziehen die Verfasser daraus nur selten Gewinn. Das hindert nicht, dass die Besitzer von Werken, die keine Aufnahme in den entsprechenden CEuvrekatalog fanden, deren Verfasser verklagen. Neben Streitfällen, in denen die Besitzer von Werken deren Aufnahme in den Katalog zu erstreiten versuchten, fehlt es auch nicht an Beispielen dafür, dass die enttäuschten Besitzer von nicht mehr als authentisch gehaltenen Werken sich auf dem Rechtsweg an den entsprechenden Experten schadlos zu halten versuchten. ${ }^{44}$ Die Gefahr, für die finanziellen Konsequenzen ihrer Gutachten haftbar gemacht zu werden, bedroht dabei nicht nur die Experten für das CEuvre einzelner Künstler, sondern alle auf dem Kunstmarkt tätigen Sachverständigen. ${ }^{45}$

Sachverständige müssen nämlich für ihre Gutachten und Expertisen haften. Diese Rechtstatsache führt dazu, dass sich zahlreiche juristische Abhandlungen mit der Bedeutung von Expertisen und den Rechtsfolgen der Gutachtertätigkeit von Kunstexperten befassen. ${ }^{46}$ In etlichen dokumentierten Fällen wurden Gut-

43 | In der Broschüre "Sachverständigenwesen der IHK Region Stuttgart: Rechtliche Grundlagen " vom Dezember 2012 heißt es dazu in § 8, Abs. 4: "Der Sachverständige hat bei der Erbringung seiner Leistung stets darauf zu achten, dass er sich nicht der Besorgnis der Befangenheit aussetzt. (...) Insbesondere darf der Sachverständige nicht (...) Gegenstände erwerben oder zum Erwerb vermitteln, (...) es sei denn, er erhält den entsprechenden Folgeauftrag nach Beendigung des Gutachtenauftrags."

44 | Brühl 2008 (wie Anm. 12), S. 66-72.

45 | Zu diesem Problem vgl. Nakov, Andrei: Das Kunstwerk: Ein Produkt wie andere, in: Süddeutsche Zeitung, 22. 06.2013, S. 16.

46 | Vgl. Brühl 2008 (wie Anm. 12); Wolf, Andreas: Die Expertise. Inhalt, Form und Rechtswirkungen von Kunstgutachten, Dissertation, Bonn 2011; Heuningen-Huene Gerrick von: Zur Rechtsstellung und Haftung des Kunstsachverständigen, in: Heinz-Peter Mansel u.a. (Hg.): Festschrift für Erik Jayme, München 2004, S. 1461-1474; Simons, Simone: Fachliche Autorität im Kunsthandel und ihre haftungsrechtliche Bedeutung, Dissertation, Konstanz 1999; Gerlach, Thilo: Die Haftung für fehlerhafte Kunstexpertisen (Schriftenreihe des Archivs für Urheber- und Medienrecht, 156), Baden-Baden 1998; zusammenfassend: Hoeren, Thomas u.a. (Hg.): Handbuch Kunst und Recht (Schriftenreihe zum Urheber- und Kunstrecht, 7), Frankfurt a.M. u.a. 2008, S. 198-215; Ebling, Klaus (Hg.): Kunstrecht, München 2007, S. 242-245. 
achter und Sachverständige für ihre Gutachten und Expertisen haftbar gemacht. Bei Gutachten beschränkt der Sachverständige seine Haftung auf den Besteller, während einer Expertise diese Haftungsbeschränkung fehlt. Es gilt hier die allgemeine Expertenregelhaftung, so dass die Haftung auch gegenüber all denen gilt, denen gegenüber sein Auftraggeber von dem Gutachten Gebrauch macht. Teure Prozesse und hohe Anwaltshonorare haben in den USA dazu geführt, dass Expertenkomitees ihre Arbeit niedergelegt haben. ${ }^{47}$

\section{FAZIT}

Die Rolle von Experten und Kunstsachverständigen auf dem Kunstmarkt ist so schillernd wie vielfältig. Trotz einer zunehmenden Zahl von Methoden und Möglichkeiten der kunsttechnologischen Analyse von Werken der Bildenden Kunst bleibt das Vertrauen in Kennerschaft und Expertise für die Wertbildung unerlässlich. Bedeutsam für die Zuschreibung eines Kunstwerkes und deren Glaubwürdigkeit ist nicht allein das objektiv argumentierende Urteil eines Experten, sondern zugleich dessen Unabhängigkeit. Sie ist bei den allein aus wissenschaftlichem Interesse forschenden Kunsthistorikern an Universitäten und Museen gegeben, die intrinsisch motiviert sind und nicht wirtschaftlich handeln. Bei einem auf Honorarbasis arbeitenden Gutachter kann sie nur bei beruflicher Selbständigkeit bestehen, denn nur dieser Status gewährleistet die Unabhängigkeit von Weisungen Dritter. Im Verhältnis zum Auftraggeber stellt er allerdings nur dann eine Garantie der inneren und äußeren Unabhängigkeit dar, wenn keine Geschäftsinteressen verfolgt werden, die über die Wahrnehmung eines Gutachten- oder Beratungsauftrages hinausgehen. ${ }^{48}$

Darüber hinaus wäre es wünschenswert, dass der Handel von Kunst und das Sammeln nicht mehr primär wirtschaftlichen Interessen folgten. Denn wer aus Leidenschaft und innerem Antrieb Kunst kauft und sammelt, wird ganz nebenbei eine Sachkenntnis erwerben, die irgendwann schriftliche Wertgutachten obsolet werden lässt. Und fraglos wird es zu den ersten Erkenntnissen des selbstbewussten Sammlers gehören, dass ein Kunstwerk, dem ausdrücklich eine Expertise beigegeben ist, diese oft auch nötig hat.

47 | Nakov (wie Anm. 45).

48 | Es wäre zu wünschen, dass dieses vom Verband unabhängiger Kunstsachverständiger e.V. (VUKS) in der Vereinssatzung festgeschriebene Ideal allen Kunstsachverständigen zur Verpflichtung würde. URL: www.vuks.de/statuten.html (10.07.2013). 